\title{
Digital storage and transmission of cardiovascular images: what are the costs, benefits and timetable for conversion?
}

\author{
James D Thomas, Steven E Nissen
}

A new word has recently appeared in the lexicon of computers, the pedabyte, which refers to a digital storage unit of 1 quadrillion (1000000000000 000) bytes of information. For those of us who remember the Neanderthal era of digital storage using punch cards (1970s) or floppy disks (early 1980s), this number, representing a million gigabytes, is truly staggering. Nevertheless, it is approximately the magnitude of digital storage needed to record one year's worth of echocardiographic and angiographic data from the Cleveland Clinic Heart Center. Despite the daunting challenges, there remains little doubt that digital storage of all cardiovascular imaging studies will become feasible over the next several years. Indeed, many authorities confidently predict that a decade from now, digital imaging will be the main method used to store all medical images. What forces are driving this massive conversion from analogue to digital storage? What advantages will digital imaging bring to the cardiovascular practitioner and how much will such systems cost? How can such an enormous quantity of data be stored, retrieved, and transmitted around a hospital? This review will try to answer the "why", "how", and (perhaps most importantly) "when" of digital cardiac image storage.

\section{Principles of digital imaging}

Digital imaging encodes the grey scale or colours within an image as a series of numbers, each of which describe the grey value or hue of a small subunit of the image known as a picture element (pixel). The resolution of the digital image is determined by the number of pixels, typically ranging from 256 rows by 256 columns (64000 pixels) to 1024 by 1024 (1 million pixels). For grey scale images (angiography), devices typically assign 8 bits per pixel (256 levels) whereas colour imaging devices provide up to 24 bits ( 16.7 million hues). For moving images, the requirement to generate several frames each second adds rapidly to the magnitude of the storage requirements. Echocardiography typically requires a 512 by 512 pixel matrix with 24 bit colour at 30 frames per second ( 24 megabytes per second), while angiography (at 8 bits per pixel) requires "only" about 7.5 megabytes per second.

\author{
Why store cardiovascular images \\ digitally? \\ IMAGE FIDELITY
}

From the figures provided, it is apparent that digital imaging can quickly generate enormous quantities of data. Given these massive storage requirements, why should we seek to archive images using digital methods? The advantages of digital storage and transmission of cardiovascular images are many, offering the potential to radically change current paradigms of patient care. ${ }^{1}$ For the various imaging methods (echocardiography, angiography, and nuclear), the issues are somewhat different, but the principles remain the same. Because images are encoded as a series of numbers, the precise original image can be reconstructed whenever and wherever required. As long as the numerical data are retained, digital storage does not result in any degradation of image quality. This feature contrasts with analogue methods such as videotape, where image storage results in a reduction in resolution and the addition of random noise. The differences between analogue and digital storage are most noticeable when images are duplicated. Each generation of videotape exhibits progressive deterioration in image quality, whereas each duplicate copy of a digitally recorded study is identical to the original.

\section{IMAGE DISPLAY}

Digitally stored images are more readily displayed and more easily reviewed than their analogue counterparts. In the catheterisation laboratory, digital angiography enables virtually instantaneous replay of high quality images with random access to any injection sequence. During angioplasty, this instantaneous review capability enables evaluation of the results of each intervention before proceeding with the next. Complications such as intraluminal thrombus and dissection can be rapidly identified and appropriate action initiated. Injection sequences can be reviewed in a continuous cine-loop at virtually any replay speed with close examination of a magnified portion the image to study the structural details (zooming). Digital image enhancement (convolution filtration) can sharpen or otherwise improve image quality. Similar benefits are evident in echocardiography, where all images from a study may be reviewed ran- 
domly and instantaneously without the inconvenience and image degradation associated with videotape storage. In stress echocardiography, side-by-side comparison of rest and stress images with display optimisation (contrast and brightness adjustment, pseudocolourisation, and correction for translation) can only be achieved with digital storage.

NETWORKING AND TRANSMISSION

Improvements in high-speed computer networks within medical centres and throughout society (the Internet) will enable transmission of digital stored images throughout hospitals or between institutions. When such networks have fully evolved, digitally stored images will be viewable throughout the hospital, both by cardiologists and primary care physicians. This enhanced access to images has the potential to greatly improve patient care by enabling all involved parties to share common information. Furthermore, prior studies will be readily available for comparison, enabling serial comparisons to evaluate the impact of changes in treatment or intercurrent events. Digital images can also be transmitted from hospital to hospital, allowing for remote consultation and referral of patients. ${ }^{2}$

\section{Quantitative analysis}

Because digital images are stored in a format readily accessible by computers, quantitative image analysis is greatly facilitated. With analogue storage methods, images must be "digitised"-converted into a digital computer format for quantitative analysis. The most salient example is quantitative coronary angiography in which cineangiographic films are digitised to enable of the dimensions of the lumen to be measured. The additional steps required to perform this analysis using film are cumbersome and costly and introduce sources of error in measurements. Similarly, digital echocardiographic files can have built in calibration factors, facilitating the quantification of ventricular volume, mass, and ejection fraction. Furthermore, Doppler flow maps may have the actual velocities encoded (rather than simply a colour image) enabling automated quantification of cardiac output ${ }^{3}$ and valvar regurgitation. ${ }^{4}$

\section{Health care costs}

Finally, digital storage has the potential to significantly reduce healthcare costs. In angiography, $35 \mathrm{~mm}$ film is costly, averaging about $\$ 75$ $(£ 50)$ per patient for processing and storage. Eventually, the expense of digital storage of data required for cardiac catheterisation (about one gigabyte) will decline to a few dollars, perhaps even a few cents. The cost advantages are less obvious for echocardiography, primarily because analogue videotape is so inexpensive. Nonetheless, its seems likely that technical progress will eventually enable digital storage of ultrasound to compete economically with videotape. In echocardiography there remain many other cost advantages for digital storage, including elimination of duplicate studies and reduction in the cardiologist's time for reading and interpreting ultrasound studies. Virtually every practitioner has experienced the consternation of looking for a particular echocardiographic study within a library of thousands of tapes. Once located, the requirement to fast forward to the images of interest further delays the review process. This inefficiency of image retrieval from tape archives must be viewed as part of the economic costs of analogue systems.

\section{Evolution of storage technology}

The computer technology enabling large-scale storage of digital images is evolving rapidly. Since 1960 , the computer industry has witnessed a doubling in the ratio of performance to price every 18 months, a trend that, if anything, seems to be accelerating (Moore's law). Indeed, if the same progress had been made in the automotive industry, the price of an average automobile today would be less than 2 cents $(1 \mathrm{p})$. Most critical to a digital archival and retrieval system is a large (actually massive) integrated set of digital storage devices. The heart of such a system might consist of a large digital tape jukebox providing many terabytes (trillions of bytes) of storage for an entire hospital. Since the response time of such a system might require several minutes to retrieve a particular study, most authorities envisage a hierarchical stucture to hospitalwide digital storage systems. Thus the slower tape jukebox would be complemented by an a faster array of hard disks encompassing several hundred gigabytes. Other storage capabilities might be distributed (decentralised) to individual laboratories such as those for echocardiography and catheterisation, but available to others via a network. Future technological developments may allow the holographic storage of terabyte quantities of imaging data on a CD-ROM sized disc. ${ }^{5}$ In a jukebox such systems could easily reach the ethereal pedabyte in capacity.

\section{Evolution of network technology}

The all-digital hospital will place great demands on computer networks. To deliver digital images throughout the hospital will require a very fast network, requiring evolution from the current Ethernet standard (10 megabits per second, Mbps) to fibreoptic Fast Ethernet speeds (100 Mbps) or to the even more spritely Asynchronous Transfer Mode ATM (155 Mbps to > 500 Mbps). Governments and private companies are currently making immense investments to ensure that such high-speed links are widely available. Development of sophisticated software to track the storage location of the many thousands of examinations performed annually represents another critical step toward to making digital storage a practical reality in hospitals. Such software must upload images from near-line archival storage to on-line hard disk storage. Ideally such systems would function 
automatically, so that when a patient is admitted or has an outpatient examination scheduled, relevant digital imaging studies would be retrieved automatically from archival storage.

\section{The standards problem}

Despite the enormous potential for digital imaging, a singular daunting challenge stands in the way of the mass conversion from analogue to digital cardiovascular imaging. This problem can be summarised in a a single word-standardisation. Until recently, commercial digital image archiving systems for medical applications typically employed proprietary, closed technology for data storage. Accordingly, a study recorded using one manufacturer's system could not be viewed on another vendor's equipment. The lack of compatibility between digital archiving systems was described in a position statement of the American College of Cardiology as the "tower of Babel" in cardiovascular medicine. These dangers are reminiscent of the competition during the 1980s between the Betamax and VHS videotape cassette formats. In the case of videotape, the absence of an industry-wide standard cost consumers billions of dollars and exasperated a generation of echocardiographers who were forced to maintain the capability to review both formats.

\section{The DICOM Standard Committees}

Faced with the disaster of a proliferation of non-standardised digital image formats, physicians from around the world have to joined together to develop worldwide compatibility standards for image interchange. In 1992 the American College of Cardiology (ACC) assembled interested parties to attempt to coordinate the angiographic standard. For cardiac ultrasound, physicians appointed by the American Society of Echocardiography (ASE) also met with industry representatives. These working groups included echocardiographers and angiographers appointed by the European Society of Cardiology (ESC). Initial meetings led to an agreement by manufacturers, through the National Electrical Manufacturers Association (NEMA), and all three medical organisations (ACC, ASE, and ESC) to work cooperatively towards the development of suitable standards. These organisations coordinated participation through an existing effort known as DICOM (Digital Imaging and Communications in Medicine), a group actively developing digital imaging standards for the entirety of medicine. Since 1992 the ad hoc committees have met two days a month in Washington, DC to work out details of an interchange standard. The echocardiography standards effort for the ASE is chaired by one of us (JT) while the angiographic committee is co-chaired by the other $(\mathrm{SN})$

Scope of the angiographic standard

Because of the multiplicity of roles served by cineangiographic film, the angiographic com- mittee focused on solving the one critical problem facing digital cardiac catheterisation-development of a format and media for interchange of angiographic studies. The committee deliberately avoided any attempt to standardise the acquisition, display, or archiving of angiographic images. After lengthy discussion and debate, the recordable 5.25 inch optical compact disk (CD-R) emerged as the optimal interchange medium for angiography. The medium is physically similar to the CDROM used in popular multimedia applications and has a capacity of about 680 megabytes. The exchange standard for cardiac angiography is nearly completed and was demonstrated in 1995 at the both ACC Scientific Sessions (termed DISC '95) and the annual meeting of the ESC. This format has now been adopted by all major catheterisation laboratory vendors and is beginning to appear in end-user products. ${ }^{6}$

\section{Scope of the ultrasound standard}

In echocardiography the needs for image interchange are more diverse. ${ }^{7}$ Accordingly several interchange media are supported by the standard. Grey-scale, colour, and spectral Doppler images can be exchanged over a network or stored on 1.44 megabyte floppy disks, 3.5 and $5 \cdot 25$ inch magneto-optical drives, and CD-R disks. Calibration factors may be stored for linear, temporal, and velocity measurements and three-dimensional registration. ${ }^{8}$ This standard was recently demonstrated at meetings of the American Society of Echocardiography and the European Congress of Cardiology, with programming by the International Consortium for Medical Imaging Technology. Such demonstrations are important for solidifying support for the standard, identifying any ambiguities in its codification, and educating the medical consumers to the importance of DICOM. With the approval of a DICOM disk storage format for nuclear medicine, demonstrations (at the 1996 ACC Scientific Sessions) showed true multimodality storage, dramatically illustrating the potential for a patient to carry all his or her medical data, including images, on a disk.

\section{Image compression: angiography} LOSSLESS METHODS

The CD-R storage capacity of $680 \mathrm{MB}$ permits recording of approximately 2400 frames per disk. This capacity is insufficient to record all cardiovascular examinations on a single piece of media, which can reach 4000 images or more. Accordingly, the Ad Hoc Standards Committee used digital data compression to increase the storage capacity of the CD-R. The compression scheme adopted by the committee is termed "lossless" because decompressed images are identical to the original uncompressed images. This lossless method results in an approximately twofold reduction in the amount of storage space required for each image frame (increasing $C D$ capacity to about 4800 frames of angiogra- 
phy). Methods termed "lossy" result in much greater data compression (typically 6:1 to 25:1) but result in some reduction in image quality. The Ad Hoc Angiographic Committee did not include lossy compression for image storage on CD-R, because the clinical utility of a suitable compression scheme and acceptable compression ratio had not been sufficiently determined.

\section{Compression and performance \\ limitations}

The DISC '95 demonstration emphasised accurate and complete exchange of images, not necessarily full-motion display of images. The data transfer rates available using CD-R limited the performance of the CD-R medium in this initial demonstration. This technical limitation allowed display of angiographic or echocardiographic images directly from the CD-R at no more than 7.5 frames per second at the DISC'95 demonstration. Full-motion display and review of DICOM images using currently available $\mathrm{CD}$ drives requires "uploading" of images from the exchange medium to RAM or a computer hard-disk. Although future developments will gradually increase recording and playback performance of $C D-R$, drive speeds sufficient for viewing at 30 frames per second directly from the medium are unlikely in the immediate future. Because of the performance limits of CD-R, several equipment vendors demonstrated an alternative approach using "lossy" compression at the 1995 ACC meeting. By using greater compression ratios on this proprietary $\mathrm{CD}$, the retrieval speed is enhanced to 30 frames per second, but with some degree of image degradation. It is important for consumers to understand that this "lossy" approach to image compression was not part of the DISC '95 demonstration and does not yet represent an official component of the standard.

\section{The compression study: angiography}

In evaluating appropriate methods for data compression, the Ad Hoc Angiographic Working Group did not rule out the use of "lossy" compression if clinical studies demonstrated no significant loss of diagnostic image quality. Therefore, the ACC and industry have initiated a clinical study of "lossy" compression to determine whether this approach is suitable for inclusion as a part of the DICOM standard. This complex study will examine the ability of observers to perform the subtle detection tasks required to interpret angiographic images. The results of this study should be available by late in 1996 .

\section{Image compression: echocardiography} JPEG ALGORITHM

Despite the dramatic gains in computer storage, it is still impractical to store and transmit complete echocardiographic studies (approximately 20 gigabytes for a 10 minute study).
While considerable savings can be obtained by storing only single cardiac cycles from each echocardiographic view and using a quadscreen display where practical, ${ }^{9}$ further digital compression is important. The Ultrasound DICOM Standard supports the use of the "Packbits" run length encoding algorithm, achieving $3: 1$ to $7: 1$ compression in a completely lossless fashion. In addition, the ultrasound standard allows the use of the JPEG (Joint Photographic Expert Group) algorithm for "lossy" digital image compression. ${ }^{10}$ Objective and subjective assessments of echocardiographic image and diagnostic quality have shown that the JPEG algorithm can compress these files at least 20 fold without noticeable degradation. ${ }^{11-13}$ Unlike angiography, lossy compression is much more intuitively acceptable in echocardiography because current methods (videotape) are "lossy." Indeed, storage on Super-VHS video tape is associated with image degradation approximately equivalent to $26: 1 \mathrm{JPEG}$ compression.

\section{MPEG ALGORITHM}

It should be noted that JPEG operates on each image frame individually, eliminating redundant information. Much higher compression ratios are possible if the approach takes advantage of interframe redundancy. Algorithms such as MPEG (Motion Picture Experts Group) and H.261 (used in video conferencing) allow compression ratios greater than 100:1 with acceptable image quality for many applications. Higher levels of compression will enable the transmission of realtime echocardiographic images over high speed telephone lines such as T1 $(1.54 \mathrm{Mbps})$ or even fractional T1 (384 kbps), permitting remote consultation from througout the world. If such compression algorithms are clinically validated, they will likely be codified within the DICOM standard.

\section{Conclusion and recommendations NEED FOR STANDARDS}

We stand on the threshold of an era of all digital storage and transmission of cardiovascular images-a transition that will profoundly affect our profession. For the most part, cardiologists will simply ride along with the tidal wave of digital technology that is moving rapidly through the entertainment and telecommunication fields. We must leverage off these enormous markets to achieve cost efficacy in digital medical imaging. Indeed, had the videotape recorder been developed and marketed only for echocardiography, it might cost $\$ 25000$ ( 116000$)$ per unit. With computer technology changing so rapidly, how can the medical consumer intelligently understand and participate in this exciting field? The key words are standards (DICOM) and openness in system architecture. To assure interoperability with other vendors' equipment within the hospital and colleagues at other hospitals, it is essential that all equipment utilises the DICOM standard for both network and media interchange. 
Conversion strategy: conformance

In developing a conversion strategy, consumers must realise that DICOM is specialised for many different modalities, and support for all of them will not be expected in most equipment (for example, a CT scanner would have little need to display echocardiograms). To specify precisely which aspects of DICOM are supported, vendors publish conformance claims, which should be studied carefully (most probably in consultation with a trusted adviser) to assure that a given piece of equipment will meet the customer's need. An open architecture is critical to taking advantage of the ongoing computer revolution. One cannot expect any computer device to remain state-of-the-art for very long. Accordingly, an upgrade path-that does not lock the consumer into a single vendors' proprietary hardware-will be important to assure continuing cost competitiveness.

\section{Timetable and conclusions}

Exactly when the rising benefits of digital storage overtake the falling costs of the technology for a given facility or image modality will depend on many factors, such as the underlying network architecture and the efficiency of the current film, video, and tape-based solutions. Nevertheless, we can expect that over the next 10 years, medicine will reach the point where digital storage and retrieval of cardiovascular images are not only possible, but regarded as the standard of care in a modern medical facility.

1 Thomas JD. Digital storage of echocardiograms. Coronary Artery Disease 1995;6:4-9.

2 Sobczyk WL, Solinger RE, Rees AH, Elbl F. Transtelephonic echocardiography: successful use in a tertiary pediatric referral center.

3 Sun, JP, Stewart WJ, Fouad FM, Christian R, Klein AL, Thomas JD. Automated flow measurement using spatial (abstract). Circulation 1995;92:I-14.

4 Vandervoort PM, Thoreau DH, Rivera JM, Levine RA, Weyman AE, Thomas JD. Automated flow rate calculations based on digital analysis of flow rate convergence proximal to regurgitant orifices. $\mathcal{F} \mathrm{Am}$ Coll Cardiol 1993; proximal to

5 Heanue JF, Bashaw MC, Hesselink L. Volume holographic storage and retrieval of digital data. Science 1994;265 749-52

6 Nissen SE, Pepine CJ, Bashore TM, Block PC, Bonchek LI, Brinker JA, et al. Cardiac angiography without cine film: erecting a "tower of Babel" in the cardaic catheterization laboratory (American College of Cardiology position statement). $\mathcal{f}$ Am Coll Cardiol 1994; 24:834-7.

7 Thomas JD, Khandheria B. Digital formatting standards in medical imaging: a primer for echocardiographers. $\mathcal{f} \mathrm{Am}$ Soc Echocardiogr 1994;7:100-4.

8 Thomas JD. The DICOM image formatting standard: what it means for echocardiographers. If $\mathrm{Am}$ Soc Echocardiogr 1995;8:319-27.

9 Feigenbaum H. Practical approach to a digital echocardio-

10 Leger A, Omachi T, Wallace GK. JPEG still picture compression algorithm. Optical Engineering 1991;30:947-54.

11 Karson TH, Chandra S, Morehead A, Nissen SE, Thomas Karson TH, Chandra S, Morehead A, Nissen SE, Thomas
JD. Digital compression of echocardiographic images: is it viable? Computers in Cardiology 1993;831-34.

12 Karson TH, Chandra S, Morehead A, Stewart WJ, Nissen SE, Thomas JD. JPEG compression of digital echocardiographic images: impact on image quality. $\mathcal{f} \mathrm{Am}$ Soc diographic images: impact on

13 Karson TH, Zepp RC, Chandra S, Morehead A, Thomas JD. Digital storage of echocardiograms offers superior image quality to analog storage even with $20: 1$ digital compression: Results of the Digital ERA (Echo Record Access) study. $₹ \mathrm{Am}$ Soc Echocardiogr 1996 (in press). 